\title{
Improved timing formula for the PSR B1257+12 planetary system
}

\author{
Maciej Konacki ${ }^{1}$, Andrzej J. Maciejewski ${ }^{2}$ \\ Toruń Centre for Astronomy, Nicolaus Copernicus University, \\ 87-100 Toruń, Gagarina 11, Poland \\ Alex Wolszczan ${ }^{3}$ \\ Department of Astronomy and Astrophysics, Penn State University \\ University Park, PA 16802, USA \\ Toruń Centre for Astronomy, Nicolaus Copernicus University \\ ul. Gagarina 11, 87-100 Toruń, Poland
}

\begin{abstract}
We present a new analysis of the dynamics of the planetary system around the pulsar B1257+12. A semi-analytical theory of perturbation between terrestrial-mass planets $\mathrm{B}$ and $\mathrm{C}$ is developed and applied to improve multi-orbit timing formula for this object. We use numerical simulations of the pulse arrival times for PSR B1257+12 to demonstrate that our new timing model can serve as a toll to determine the masses of the two planets.
\end{abstract}

Subject headings: celestial mechanics — planetary systems — pulsars: individual (PSR $\mathrm{B} 1257+12)$

\section{Introduction}

The first extra-solar planetary system has been discovered around a millisecond pulsar B1257+12 (Wolszczan \& Frail 1992). The system consists of three planets named $\mathcal{A}, \mathcal{B}$ and $\mathcal{C}$ with planets $\mathcal{B}$ and $\mathcal{C}$ having the orbits close to a 3:2 commensurability. This circumstance allows us to analyze the dynamics of the system beyond the classical Keplerian approximation. Namely, in such configuration, the gravitational interactions of planets $\mathcal{B}$ and $\mathcal{C}$ give rise to observable time variations of $\mathcal{B}$ and $\mathcal{C}$ orbital elements. It was thoroughly discussed by Rasio et al. (1992) and Malhotra (1993a) (see also Malhotra et al. 1992; Malhotra 1993b; Rasio et al. 1993; Peale 1993). Subsequently, these studies were used to confirm the existence of the PSR B1257+12 planetary system through

\footnotetext{
${ }^{1}$ e-mail: kmc@astri.uni.torun.pl

2e-mail: maciejka@astri.uni.torun.pl

${ }^{3}$ e-mail: alex@astro.psu.edu
} 
detecting, in the timing observations of the pulsar, the presence of those non-keplerian variations (Wolszczan 1994)

However, the application of non-keplerian dynamics goes further than the confirmation of the discovery. It can be used to derive some interesting information about the system which is not otherwise accessible. The aim of this paper is to apply the theory of perturbed planetary motion and derive an improved model for the timing observations of PSR B1257+12. Such model, as we show on simulations, should lead to determination of the masses of planets $\mathcal{B}$ and $\mathcal{C}$, as well as the inclinations of their orbits. To this end, in section 2 we analyze the equations of motion in the barycentric and Jacobi coordinate systems, which we use in the paper. In section 3 we show how these two slightly different representations are related to the commonly used timing model for pulsars with companions. In section 4 we demonstrate how to express such timing formula in terms of the osculating orbital elements. In section 5 we show how to obtain the osculating elements of $\mathcal{B}$ and $\mathcal{C}$. In section 6 we present an improved timing model describing the motion of this system and finally, in section 7 , we perform numerical tests which show how it can be used in practice.

\section{Equations of Motion}

Let us consider a system consisting of a neutron star $P_{0}$ with the mass $m_{0}$, and $N$ planets $P_{i}$ with masses $m_{i}$. In an arbitrary inertial reference frame equations of motion of this system have the form

$$
m_{i} \ddot{\mathbf{R}}_{i}=-G \sum_{\substack{j=0 \\ j \neq i}}^{N} \frac{m_{i} m_{j}}{R_{i j}^{3}}\left(\mathbf{R}_{i}-\mathbf{R}_{j}\right)
$$

where

$$
R_{i j}=\left\|\mathbf{R}_{i}-\mathbf{R}_{j}\right\|, \quad i, j=0, \ldots, N,
$$

and $G$ is the gravitational constant. The Hamiltonian function for system (1) has the form

$$
H=\frac{1}{2} \sum_{i=0}^{N} \frac{1}{m_{i}} \mathbf{P}_{i}^{2}-\sum_{0 \leq i<j \leq N} \frac{G m_{i} m_{j}}{R_{i j}},
$$

and equations (1) can be written as Hamilton's equations

$$
\frac{d}{d t} \mathbf{R}_{i}=\frac{\partial H}{\partial \mathbf{P}_{i}}, \quad \frac{d}{d t} \mathbf{P}_{i}=-\frac{\partial H}{\partial \mathbf{R}_{i}}, \quad i=0, \ldots, N
$$

Analytical perturbation theory for a planetary system is usually formulated in the so-called Jacobi coordinates $\mathbf{r}_{i}, i=0, \ldots, N$ which are defined in the following way

$$
\mathbf{r}_{k}=\mathbf{R}_{k}-\frac{1}{\mu_{k-1}} \sum_{i=0}^{k-1} m_{i} \mathbf{R}_{i}, \quad \text { for } \quad k=1, \ldots, N,
$$


and

$$
\mathbf{r}_{0}=\frac{1}{\mu_{N}} \sum_{i=0}^{N} m_{i} \mathbf{R}_{i}, \quad \mu_{k}=\sum_{i=0}^{k} m_{i} .
$$

In other words, $\mathbf{r}_{i}$ is the radius vector from the center of mass of bodies $P_{0}, \ldots, P_{i-1}$ to body $P_{i}$, and $\mathbf{r}_{0}$ is the center of mass of the system. The above formulae define a one-to-one relationship between Cartesian inertial coordinates and Jacobi coordinates. The inverse relationship has the form

$$
\mathbf{R}_{k}=\mathbf{r}_{0}+\frac{\mu_{k-1}}{\mu_{k}} \mathbf{r}_{k}-\sum_{i=k+1}^{N} \frac{m_{i}}{\mu_{i}} \mathbf{r}_{i}, \quad k=0, \ldots, N,
$$

where we assume $\mu_{-1}=0$. In terms of canonical Jacobi coordinates, Hamiltonian (2) reads

$$
\begin{aligned}
H= & \frac{1}{\mu_{N}} \mathbf{p}_{0}^{2}+\sum_{i=1}^{N} \frac{\mu_{i}}{\mu_{i-1} m_{i}} \mathbf{p}_{i}^{2}-G \sum_{i=1}^{N} \frac{m_{0} m_{i}}{r_{i}}+ \\
& +\tilde{H}_{1}\left(\mathbf{r}_{1}, \ldots, \mathbf{r}_{N}\right),
\end{aligned}
$$

where $\tilde{H}_{1}=\tilde{H}_{1}\left(\mathbf{r}_{1}, \ldots, \mathbf{r}_{N}\right)$ is its perturbative part. If we assume that masses of planets are small and all are of the same order $\epsilon$, then $\tilde{H}_{1}$ is of order $\epsilon^{2}$, i.e., $\tilde{H}_{1}=H_{1}+\mathcal{O}\left(\epsilon^{3}\right)$, where

$$
H_{1}=-G \sum_{1 \leq i<j \leq N} m_{i} m_{j}\left[\frac{1}{r_{i j}}-\frac{\mathbf{r}_{i} \cdot \mathbf{r}_{j}}{r_{i}}\right] \text {. }
$$

From the form of Hamiltonian (7) it follows that $\mathbf{p}_{0}$ is a first integral and that the equations for variables $\left\{\mathbf{r}_{1}, \ldots, \mathbf{r}_{N}, \mathbf{p}_{1}, \ldots, \mathbf{p}_{N}\right\}$ do not depend on its particular value. Thus, we can assume that $\mathbf{p}_{0}=\mathbf{0}$ implying that $\mathbf{r}_{0}$ is constant and can be set to $\mathbf{r}_{0}=\mathbf{0}$. This is equivalent to the assumption that our inertial frame is a certain barycentric reference frame. The dynamics of the system is governed by Hamilton's equations with the Hamiltonian of the form

$$
H=H_{0}+H_{1}+\cdots
$$

where

$$
H_{0}=\sum_{i=1}^{N} \frac{\mu_{i}}{\mu_{i-1} m_{i}} \mathbf{p}_{i}^{2}-G \sum_{i=1}^{N} \frac{m_{0} m_{i}}{r_{i}}
$$

is the unperturbed part of the Hamiltonian describing a system of $N$ independent planets. It follows from the form of Eq. (10) that each planet moves in a Keplerian orbit in the same way as a body with the mass $m_{i} \mu_{i-1} / \mu_{i}$ around a fixed gravitational center $m_{0} m_{i}$. Each of these Keplerian motions can be parameterized by Keplerian elements $\left\{T_{p}, a, e, i, \omega, \Omega\right\}$ - the time of pericenter, semimajor axis, eccentricity, inclination, argument of pericenter and the longitude of ascending node, respectively. 


\section{Timing model and coordinate systems}

Timing observations of pulsars represent measurements of the times of arrival of pulsars pulses (TOAs). An extraordinary precision of timing measurements allows a detection of very low-level effects in timing residuals (see for review Lyne \& Graham-Smith 1998). In the case of a binary pulsar the observed TOAs exhibit periodic variations resulting from the motion of the pulsar around the center of mass. Such variations are modeled with the formula

$$
\Delta t=x\left[(\cos E-e) \sin \omega+\sqrt{1-e^{2}} \sin E \cos \omega\right],
$$

where

$$
x=a \sin i / c, \quad E-e \sin E=n\left(t-T_{p}\right), \quad n=\frac{2 \pi}{P},
$$

and $\Delta t$ is the additional delay/advance in TOAs, $E$ is eccentric anomaly, $a, e, \omega, \sin i, P, T_{p}$ are Keplerian elements of the orbit and $c$ is the speed of light. When a pulsar has $N$ planets the TOA variations become

$$
\begin{aligned}
& \Delta t=\sum_{j=1}^{N} x_{j} {\left[\left(\cos E_{j}-e_{j}\right) \sin \omega_{j}+\right.} \\
&\left.\left.+\sqrt{1-e_{j}^{2}} \sin E_{j} \cos \omega_{j}\right)\right] .
\end{aligned}
$$

Note that in practice the parameters of the model which we determine, by means of the least-squares fit to the data, are $x_{j}, e_{j}, \omega_{j}, P_{j}, T_{p j}$, i.e., the projection of semi-major axis of the pulsar's orbit, eccentricity, argument of pericenter, orbital period and time of pericenter. In order to precisely understand and interpret these parameters we describe the pulsar's motion in the barycentric reference frame with the $z$-axis of the system directed toward the barycenter of the solar system and $x y$ plane of the reference frame in the plane of the sky. This way, we have

$$
\Delta t=-\frac{1}{c} \mathbf{R}_{0} \cdot \widehat{\mathbf{Z}}, \quad \mathbf{R}_{0}=-\frac{1}{m_{0}} \sum_{i=1}^{N} m_{i} \mathbf{R}_{i}
$$

where $\widehat{\mathbf{Z}}$ is the unit vector along the $z$-th axis of the pulsar system barycentric frame and $\mathbf{R}_{j}$ are barycentric positions of planets. Assuming that

$$
\frac{m_{j}}{m_{0}} a_{j} \sin i_{j}=x_{j} c, \quad \frac{G m_{0}}{\left(1+m_{j} / m_{0}\right)^{2}}=n_{j}^{2} a_{j}^{3}
$$

with planets' orbital parameters $a_{j}, n_{j}, e_{j}, \omega_{j}, T_{p j}$, we can most naturally interpret the motion of the pulsar as a superposition of the elliptic motions of its planets around the barycenter of the system. However, as it was mentioned in the previous section, the analytical perturbation theory is usually formulated in Jacobi coordinates in which the TOA variations become

$$
\Delta t=-\frac{1}{c} \mathbf{R}_{0} \cdot \widehat{\mathbf{Z}}, \quad \mathbf{R}_{0}=-\sum_{j=1}^{N} \kappa_{j} \mathbf{r}_{j}, \quad \kappa_{j}=\frac{m_{j}}{\mu_{j}}
$$


where $\mathbf{r}_{j}$ are positions of planets. Furthermore, we have the following relations

$$
\kappa_{j} a_{j} \sin i_{j}=x_{j} c, \quad \frac{G m_{0} \mu_{j}}{\mu_{j-1}}=\frac{G m_{0}}{1-\kappa_{j}}=n_{j}^{2} a_{j}^{3} .
$$

Thus from the timing formula for TOA variations of a pulsar with planets it is possible to obtain two somewhat different descriptions of the pulsar motion. Although, they both represent a sum of a certain number of elliptic motions, the interpretation of some of their parameters is slightly different. Throughout the rest of this paper, we will use Jacobi coordinates as they are more convenient in the formulation of the theory of perturbed motion.

\section{Osculating orbital elements}

The non-keplerian motion of the PSR B1257+12 system can be described by means of the osculating ellipses (i.e. by means of ellipses which parameters change with time). The time evolution of orbital elements can be determined by solving the classical Lagrange's perturbation equations (see, for example Brouwer \& Clemence 1961) with the perturbation Hamiltonian given by equation (8). Such approach leads to the solution in the form

$$
\mathrm{x}=\mathrm{x}^{0}+\Delta \mathrm{x}\left(t-t_{0}\right),
$$

where $\mathrm{x}$ stands for a specific orbital element, $\mathrm{x}^{0}$ its initial value and $\Delta \mathrm{x}$ for its time dependent part of small magnitude $\Delta \mathrm{x}\left(t-t_{0}\right) / \mathrm{x}^{0} \ll 1$. In the case of the PSR B1257+12 planetary systems the most significant part of the perturbations comes from planets $\mathcal{B}$ and $\mathcal{C}$. Therefore, we can assume that the orbital elements of planet $\mathcal{A}$ are approximately constant while the elements of planets $\mathcal{B}$ and $\mathcal{C}$ change with time. Thus using the formulae for $\Delta t$ from the previous section (Eq. (12)) and assuming the time evolution of orbital elements in the form (17), the additional TOA variations $\delta t_{j}, j=\{\mathcal{B}, \mathcal{C}\}$ due to the interactions between planets $\mathcal{B}$ and $\mathcal{C}$ can be expressed as follows

$$
\begin{aligned}
\frac{c \delta t_{j}}{\kappa_{j} \sin i_{j}^{0}}= & -\Delta h_{j}\left(\frac{3}{2} a_{j}^{0}+\frac{1}{2} a_{j}^{0} \cos \left(2 \lambda_{j}^{0}\right)\right)+ \\
& +\frac{1}{2} \Delta k_{j} a_{j}^{0} \sin \left(2 \lambda_{j}^{0}\right)+ \\
& +\Delta a_{j} \sin \lambda_{j}^{0}+\Delta \lambda_{j} a_{j}^{0} \cos \lambda_{j}^{0},
\end{aligned}
$$

where

$$
\begin{aligned}
& h_{j}=e_{j} \sin \omega_{j}, \quad k_{j}=e_{j} \cos \omega_{j}, \\
& \lambda_{j}=n_{j}\left(t-T_{p j}\right)+\omega_{j},
\end{aligned}
$$

and equation (18) is given to first order in $\Delta a_{j}, \Delta \lambda_{j}, \Delta h_{j}, \Delta k_{j}$ and the lowest order in $e_{j}$ (or $h_{j}$ and $k_{j}$, since the eccentricities of planets $\mathcal{B}$ and $\mathcal{C}$ are very small). The above equations can be 
obtained from the following well-known expansions

$$
\begin{gathered}
\cos E=-\frac{e}{2}+2 \sum_{k \in \mathcal{Z}_{0}} \frac{1}{k} J_{k-1}(k e) \cos (k M), \\
\sin E=\frac{2}{e} \sum_{k \in \mathcal{Z}_{0}} \frac{1}{k} J_{k-1}(k e) \sin (k M), \quad M=\lambda-\omega
\end{gathered}
$$

where $J_{k}(x)$ is a Bessel function of the first kind of order $n$ and argument $x, \mathcal{Z}_{0}$ denotes the set of all positive and negative integers excluding zero; and an approximate relation for Bessel functions of small arguments

$$
J_{k}(x) \approx \frac{x^{k}}{2^{k} k !}, \quad x<<1
$$

Now, our next step is to find the explicit form of the functions

$$
\begin{aligned}
& a_{j}(t)=a_{j}^{0}+\Delta a_{j}(t), \quad \lambda_{j}(t)=\lambda_{j}^{0}+\Delta \lambda_{j}(t), \\
& h_{j}(t)=h_{j}^{0}+\Delta h_{j}(t), \quad k_{j}(t)=k_{j}^{0}+\Delta k_{j}(t) .
\end{aligned}
$$

\section{Semi-analytical perturbation theory}

Mutual gravitational interactions between planets cause periodic and secular changes of their orbital elements. In the case of PSR B1257+12 periodic variations can be related to conjunctions ('close encounters') of planets $\mathcal{B}$ and $\mathcal{C}$ with the frequency $n_{c e}=n_{\mathcal{B}}-n_{\mathcal{C}}$ and to the $3: 2$ nearcommensurability with the frequency $n_{r}=3 n_{\mathcal{C}}-2 n_{\mathcal{B}}$. The dynamics of this system was studied by Rasio et al. (1992) and Malhotra (1993a) (see also Malhotra 1993b; Rasio et al. 1993; Peale 1993). Malhotra (1993a) solved the Lagrange's perturbation equations for the orbital elements assuming non-resonant system with coplanar orbits. This first order perturbation theory is valid for $(\sin i)^{-1} \gtrsim 10$ (orbital inclinations larger than about 6 degrees) which corresponds to mass

ratios $m_{j} / m_{0} \gtrsim 6 \times 10^{-5}$ (and non-resonant case). When planets $\mathcal{B}$ and $\mathcal{C}$ are locked in the exact resonance it is necessary to develop a different theory of motion (Malhotra et al. 1992; Rasio et al. 1993).

It turns out that the first-order perturbation theory for this system developed by Malhotra (1993a) is not accurate enough for the purpose of determination of the planets' masses. Therefore, in this paper we present a new approach to this problem which precisely addresses the issue. Namely, first we assume that the orbits of $\mathcal{B}$ and $\mathcal{C}$ are not coplanar however their relative inclination $I$ is small. The geometry of the system can be represented as in Fig. 1 and the perturbative part of Hamiltonian expanded to the first order in $I$ and the product of the mass of planets $\mathcal{B}$ and $\mathcal{C}$, $m_{1} m_{2}$, has the following form

$$
H_{1}=-\frac{G m_{1} m_{2}}{r_{2}}\left[\left(1-2 \frac{r_{1}}{r_{2}} \cos \psi+\left(\frac{r_{1}}{r_{2}}\right)^{2}\right)^{-1 / 2}-\frac{r_{1}}{r_{2}} \cos \psi\right],
$$


where

$$
\begin{aligned}
& \psi=f_{1}+\omega_{1}-f_{2}-\omega_{2}-\tau, \quad \tau=\tau_{1}-\tau_{2}, \\
& r_{j}=\frac{a_{j}\left(1-e_{j}^{2}\right)}{1+e_{j} \cos f_{j}}, \quad j=1,2,
\end{aligned}
$$

and $f_{j}$ is the true anomaly. Note that in the above expansion, $H_{1}$ does not depend on $I$. The first term in which $I$ appears is of the second order; precisely it is $\sin ^{2}(I / 2)$. Thus as long as $\sin ^{2}(I / 2)$ is negligible, the above expansion is valid.

Next, from the classical form of Lagrange's perturbation equations (see, for example Brouwer \& Clemence 1961) we can obtain the following set of the first order differential equations for the elements $a_{j}, e_{j}, \omega_{j}, \lambda_{j}$

$$
\begin{gathered}
\dot{a}_{j}=\frac{-2}{\mu_{j} n_{j} a_{j}} \frac{\partial H_{1}}{\partial \sigma_{j}} \\
\dot{e}_{j}=\frac{1}{\mu_{j} n_{j} a_{j}^{2} e_{j}}\left[-\left(1-e_{j}^{2}\right) \frac{\partial H_{1}}{\partial \sigma_{j}}+\sqrt{1-e_{j}^{2}} \frac{\partial H_{1}}{\partial \omega_{j}}\right] \\
\dot{\omega}_{j}=\frac{-\sqrt{1-e_{j}^{2}}}{\mu_{j} n_{j} a_{j}^{2} e_{j}} \frac{\partial H_{1}}{\partial e_{j}} \\
\dot{\lambda}_{j}=n_{j}+\frac{2}{\mu_{j} n_{j} a_{j}} \frac{\partial H_{1}}{\partial a_{j}}-\frac{e_{j} \sqrt{1-e_{j}^{2}}}{\mu_{j} n_{j} a_{j}^{2}\left(1+\sqrt{1-e_{j}^{2}}\right)} \frac{\partial H_{1}}{\partial e_{j}}
\end{gathered}
$$

where $n_{j}$ is the mean motion and $\sigma_{j}$ is related to time of pericenter through $\sigma_{j}=-n_{j} T_{p j}$ so as the Kepler equation reads

$$
E_{j}-e_{j} \sin E_{j}=n_{j} t+\sigma_{j}=\lambda_{j}-\omega_{j},
$$

and the true anomaly $f_{j}$ is related to the eccentric anomaly $E_{j}$ through

$$
\tan \frac{f_{j}}{2}=\sqrt{\frac{1+e_{j}}{1-e_{j}}} \tan \frac{E_{j}}{2} .
$$

And it should be noted that the elements $\Omega_{j}, i_{j}$ remain approximately constant in the case of small relative inclinations.

In principle, such set of equations could be solved for $a_{j}, e_{j}, \omega_{j}, \lambda_{j}$. In practice however, it is extremely complicated. On the other hand, in fact we do not need analytical formulae such as those presented in Malhotra (1993a). Thus the most suitable approach is to solve this problem numerically. In order to do so we just need the explicit form of the right-hand-side functions of equations (24) which can be easily obtained using the perturbing Hamiltonian $H_{1}$. Subsequently, the equations can be solved numerically for $a_{j}, e_{j}, \omega_{j}, \lambda_{j}$. As we show in section 7 , this approach 
gives very accurate results, much more accurate than any reasonable analytical treatment. From the form of equations (24) it follows that the change of orbital parameters $\Delta \mathrm{x}$ (strictly speaking here $\Delta \mathrm{x}$ stands for $\Delta \lambda, \Delta h, \Delta k$ and $\left.\Delta a / a_{0}\right)$ is proportional to $m_{1} / m_{0}$ and $m_{2} / m_{0}$. Precisely, corrections $\Delta \mathrm{x}_{1}$ are proportional to $m_{2} / m_{0}$ and $\Delta \mathrm{x}_{2}$ are proportional to $m_{1} / m_{0}$. Let us finally note that in this model there is one additional parameter $\tau$ which in general is not known a priori. However, it can be determined through a least-squares analysis of the data.

\section{Timing formula}

We have now all the components necessary to derive a useful timing formula which will describe the motion of the PSR B1257+12 system. First of all, let us note that due to the small mass of planet $\mathcal{A}$ we will have:

$$
\begin{gathered}
\kappa_{\mathcal{A}}=\kappa_{1}=\frac{m_{\mathcal{A}}}{m_{0}} \\
\kappa_{\mathcal{B}}=\kappa_{2}=\frac{m_{\mathcal{B}}}{m_{0}+m_{\mathcal{A}}+m_{\mathcal{B}}} \approx \frac{m_{\mathcal{B}}}{m_{0}+m_{\mathcal{B}}}, \\
\kappa_{\mathcal{C}}=\kappa_{3}=\frac{m_{\mathcal{C}}}{m_{0}+m_{\mathcal{A}}+m_{\mathcal{B}}+m_{\mathcal{C}}} \approx \frac{m_{\mathcal{C}}}{m_{0}+m_{\mathcal{B}}+m_{\mathcal{C}}} .
\end{gathered}
$$

The problem of finding masses and inclinations of planets $\mathcal{B}$ and $\mathcal{C}$ can be now formulated as a least-squares problem in which we fit to the data the following function

$$
\Delta t=\Delta t_{K}(\Psi)+\delta t_{\mathcal{B}}\left(\Psi, \gamma_{\mathcal{B}}, \gamma_{\mathcal{C}}\right)+\delta t_{\mathcal{C}}\left(\Psi, \gamma_{\mathcal{B}}, \gamma_{\mathcal{C}}\right)
$$

where

$$
\begin{aligned}
& \Psi=\left\{x_{\mathcal{B}}^{0}, n_{\mathcal{B}}^{0}, e_{\mathcal{B}}^{0}, \omega_{\mathcal{B}}^{0}, T_{p \mathcal{B}}^{0}, x_{\mathcal{C}}^{0}, n_{\mathcal{C}}^{0}, e_{\mathcal{C}}^{0}, \omega_{\mathcal{C}}^{0}, T_{p \mathcal{C}}^{0}\right\}, \\
& \gamma_{\mathcal{B}}=\frac{m_{\mathcal{B}}}{m_{0}}, \quad \gamma_{\mathcal{C}}=\frac{m_{\mathcal{C}}}{m_{0}},
\end{aligned}
$$


$\Delta t_{K}(\Psi)$ describes the Keplerian part of the motion given by equation (12) and

$$
\begin{aligned}
& \delta t_{\mathcal{B}}=x_{\mathcal{B}}^{0}\left[-\Delta h_{\mathcal{B}}\left(\frac{3}{2}+\frac{1}{2} \cos \left(2 \lambda_{\mathcal{B}}^{0}\right)\right)+\right. \\
& +\frac{1}{2} \Delta k_{\mathcal{B}} \sin \left(2 \lambda_{\mathcal{B}}^{0}\right)+ \\
& \left.+\frac{\Delta a_{\mathcal{B}}}{a_{\mathcal{B}}^{0}} \sin \lambda_{\mathcal{B}}^{0}+\Delta \lambda_{\mathcal{B}} \cos \lambda_{\mathcal{B}}^{0}\right], \\
& \delta t_{\mathcal{C}}=x_{\mathcal{C}}^{0}\left[-\Delta h_{\mathcal{C}}\left(\frac{3}{2}+\frac{1}{2} \cos \left(2 \lambda_{\mathcal{C}}^{0}\right)\right)+\right. \\
& +\frac{1}{2} \Delta k_{\mathcal{C}} \sin \left(2 \lambda_{\mathcal{C}}^{0}\right)+ \\
& \left.+\frac{\Delta a_{\mathcal{C}}}{a_{\mathcal{C}}^{0}} \sin \lambda_{\mathcal{C}}^{0}+\Delta \lambda_{\mathcal{C}} \cos \lambda_{\mathcal{C}}^{0}\right],
\end{aligned}
$$

describe its non-keplerian part. In this model the initial values of osculating orbital elements replace the Keplerian elements as parameters of the model and we additionally have the parameters, $\gamma_{\mathcal{B}}$ and $\gamma_{\mathcal{C}}$, related to the masses of $\mathcal{B}$ and $\mathcal{C}$. We also need the derivatives of $\Delta t$ with respect to the parameters $\left\{\Psi, \gamma_{\mathcal{B}}, \gamma_{\mathcal{C}}\right\}$. With sufficient accuracy the derivatives with respect to $\Psi$ can be computed from the Keplerian part $\Delta t_{K}$ of the model only and the derivatives with respect to $\gamma_{\mathcal{B}}$ and $\gamma_{\mathcal{C}}$ can be easily obtained as $\delta t_{\mathcal{B}}$ and $\delta t_{\mathcal{C}}$ are proportional to them

$$
\frac{\partial \Delta t}{\partial \gamma_{\mathcal{B}}}=\frac{\delta t_{\mathcal{C}}}{\gamma_{\mathcal{B}}}, \quad \frac{\partial \Delta t}{\partial \gamma_{\mathcal{C}}}=\frac{\delta t_{\mathcal{B}}}{\gamma_{\mathcal{C}}}
$$

Now, determination of masses of $\mathcal{B}$ and $\mathcal{C}$ and inclinations $i_{\mathcal{B}}$ and $i_{\mathcal{C}}$ can be carried out in the following way. First, we assume that the mass of the pulsar $m_{0}$ is canonical (i.e. $1.4 \mathrm{M}_{\odot}$ ) and derive $m_{\mathcal{B}}, m_{\mathcal{C}}$ directly from $\gamma_{\mathcal{B}}$ and $\gamma_{\mathcal{C}}$. Subsequently inclinations can be computed from the following equations

$$
c x_{j}^{0}=\kappa_{j} a_{j}^{0} \sin i_{j}^{0}, \quad \frac{G m_{0}}{1-\kappa_{j}}=n_{j}^{0^{2}} a_{j}^{0^{3}},
$$

where $j=\{\mathcal{B}, \mathcal{C}\}$.

\section{Numerical tests and conclusions}

We start the tests with the comparison of our approach for computing osculating elements with that of Malhotra (1993a). It turns out that the most significant component of the non-keplerian motion comes from the changes of mean longitude $\lambda_{j}$. Therefore in Fig. 2 we present $\Delta \lambda_{j}$ for the case of coplanar, edge-on orbits. As we can see, the approach developed in this paper is in fact

as good as the integration of full equations of motion. For other elements we obtain very similar 
results. It should be mentioned that because the model of Malhotra (1993a) is not accurate enough in predicting the secular change of $\Delta \lambda_{j}$ and because $\Delta \lambda_{j}$ are proportional to $\gamma_{\mathcal{B}}$ and $\gamma_{\mathcal{C}}$, using such model would result in a significant error in determination of the planets' masses. Next, we compared the TOA residuals due to the non-keplerian part of the motion calculated from our model with those obtained by means of numerical integration of full equations of motion. In Fig. 3, as one can see, for small relative inclinations (Fig. 3 (a) and (b)) our model is very accurate. For larger $I$ (Fig. 3 (c) and (d)) one can see small differences but this is consistent with the assumptions made to obtain the model.

Finally, we performed the tests which show how the derived timing model can be used to obtain the masses of planets. To this end we used the TEMPO software package (see the Internet location http://pulsar.princeton. edu/tempo) modified to include our model of the non-keplerian motion of PSR B1257+12. We simulated two different sets of artificial TOAs assuming the parameters of PSR B1257+12 (Wolszczan 1994). The first set of TOAs sampled every day covered a period of 10 years. We also added Gaussian noise with $\sigma=0.1 \mu \mathrm{s}$. The second set was prepared to resemble the real timing observations of PSR B1257+12. Under such conditions we simulated TOAs for the cases described in Fig. 3. Subsequently, we applied the modified TEMPO to obtain the masses of planets $\mathcal{B}$ and $\mathcal{C}$. The results are presented in Table 1 .

The tests performed on Set 1 were used to establish a limit on applicability of our model. As one can see, for the relative inclinations $I$ of about 10 degrees the model becomes inaccurate and the relative error of determination of masses is at the level of $20 \%$. Because the relative inclination weakens the interactions of planets, using the model which assumes a small $I$, results, in such situations, in the determined masses that are smaller than the real ones. From the tests performed on Set 2 we also learn that this error is bigger than uncertainty originating in TOA measurement errors. On the other hand, when $I$ is relatively small we obtain a very accurate determination of masses thus proving that our model can be successfully applied as long as the assumptions made to derive it are satisfied.

To sum up, our model can be applied in all the cases when the 'observational' uncertainties of the planets' masses are bigger than the uncertainties resulting from the assumptions made to derive the timing model. This, in general, depends on masses of planets and the relative inclination of the orbits $I$ as well as the characteristic of the observations but one can estimate that in the case of the PSR B1257+12 data $I$ should be smaller than 10 degrees. We should also mention that for non-coplanar orbits the angle $\tau$ will be in general different from zero therefore we have to find it in order to get the proper values of the planets' masses. It can be done by computing the least-squares value of $\chi^{2}$ for a range of $\tau$ and then choosing the $\tau$ that corresponds to the minimum of $\chi^{2}$. And eventually, one has to remember that the PSR B1257+12 planetary system could be in such configuration that a more significant alteration must be done to our model. First of all, the relative inclination of the orbits could be large. In such case one would have to use the full form of the perturbative Hamiltonian $H_{1}$ which would lead to a much more complicated model with four additional parameters $\Omega_{\mathcal{B}}, \Omega_{\mathcal{C}}, i_{\mathcal{B}}, i_{\mathcal{C}}$ (instead of one $\tau$ ). Secondly, the presence of a massive distant 
planet, if significant for the theory of motion, would have to be taken into account.

\section{REFERENCES}

Brouwer, D. \& Clemence, G. M. 1961, Methods of Celestial Mechanics, (New York: Academic Press)

Lyne, A. G. \& Graham-Smith, F. 1998, Pulsar Astronomy, (New York: Cambridge University Press)

Malhotra, R., Black, D., Eck, A. \& Jackson, A. 1992, Nature, 356, 583

Malhotra, R. 1993a, ApJ, 407, 266

Malhotra, R. 1993b, ASP Conf. Ser. 36: Planets Around Pulsars, 89, (San Francisco: ASP)

Peale, S. J. 1993, AJ, 105, 1562

Rasio, F. A., Nicholson, P. D., Shapiro, S. L. \& Teukolsky, S. A. 1992, Nature, 355, 325

Rasio, F. A., Nicholson, P. D., Shapiro, S. L. \& Teukolsky, S. A. 1993, ASP Conf. Ser. 36: Planets Around Pulsars, 107, (San Francisco: ASP)

Wolszczan, A. \& Frail, D. A. 1992, Nature, 355, 145

Wolszczan, A. 1994, Science, 264, 538 
Fig. 1.- Geometry of the system. The angles $\omega_{1}, \omega_{2}$ are the arguments of pericenter, $\Omega_{1}, \Omega_{2}$ longitudes of ascending node and $i_{1}, i_{2}$ inclinations of the orbit of the planets $\mathcal{B}$ and $\mathcal{C} ; \tau_{1}$ and $\tau_{2}$ are the angles $n_{1} \mathrm{On}_{12}$ and $n_{2} \mathrm{On}_{12}$ respectively. The angles $I, \tau_{1}, \tau_{2}$ can be found by solving the spherical triangle $n_{1} n_{12} n_{2}$.

Fig. 2. - Time evolution of the mean longitude $\Delta \lambda(t)=\lambda(t)-\lambda^{0}(t)$ for the planets $\mathcal{B}$ and $\mathcal{C}$ in the case of coplanar, edge-on orbits. The solid line indicates the solution by means of the numerical integration of full equations of motion, the open circles indicate the solution obtained with the approach presented in this paper and the dash-dotted line with the model by Malhotra (1993a).

Fig. 3. - TOA residuals due to the non-keplerian part of motion of the PSR B1257+12 in four different configurations. The solution obtained by means of the numerical integration of full equations of motion is indicated with the dots and the one obtained using our model with the solid line. 
Table 1. Assumed and derived parameters of simulations ${ }^{\text {a }}$

\begin{tabular}{|c|c|c|c|c|c|c|c|c|}
\hline & \multicolumn{4}{|c|}{ Assumed } & \multicolumn{2}{|c|}{ Set 1} & \multicolumn{2}{|c|}{ Set 2} \\
\hline & $\tau$ & $I$ & $\begin{array}{c}m_{\mathcal{B}} \\
{\left[M_{\oplus}\right]}\end{array}$ & $\begin{array}{c}m_{\mathcal{C}} \\
{\left[M_{\oplus}\right]}\end{array}$ & $\begin{array}{c}m_{\mathcal{B}} \\
{\left[M_{\oplus}\right]}\end{array}$ & $\begin{array}{c}m_{\mathcal{C}} \\
{\left[M_{\oplus}\right]}\end{array}$ & $\begin{array}{c}m_{\mathcal{B}} \\
{\left[M_{\oplus}\right]}\end{array}$ & $\begin{array}{c}m_{\mathcal{C}} \\
{\left[M_{\oplus}\right]}\end{array}$ \\
\hline (a) & 0.0 & 0.0 & 3.41 & 2.83 & $3.42(1)$ & $2.82(1)$ & $3.53(51)$ & $2.63(51)$ \\
\hline (b) & 0.0 & 2.0 & 4.99 & 4.32 & $4.98(1)$ & $4.26(1)$ & $5.07(51)$ & $4.06(48)$ \\
\hline (c) & 0.0 & 10.0 & 4.82 & 4.94 & $4.06(3)$ & 4.11(3) & $4.06(51)$ & $3.79(48)$ \\
\hline (d) & 9.7 & 10.3 & 9.96 & 16.33 & $8.42(12)$ & $13.39(12)$ & $8.25(45)$ & $13.16(42)$ \\
\hline
\end{tabular}

${ }^{\text {a}}$ Figures in parentheses are $3 \sigma$ uncertainties in the last digits quoted. 


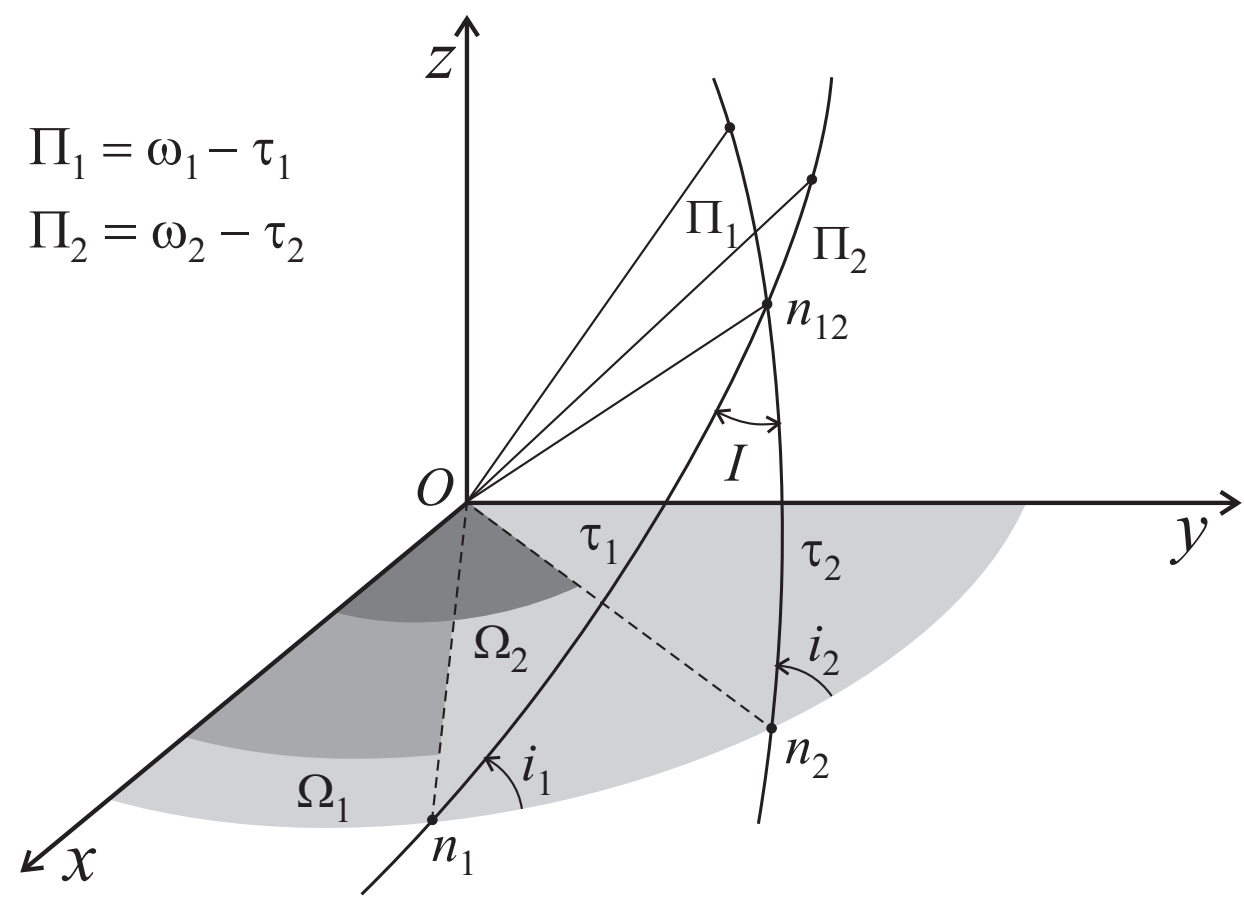

Fig. 1.-

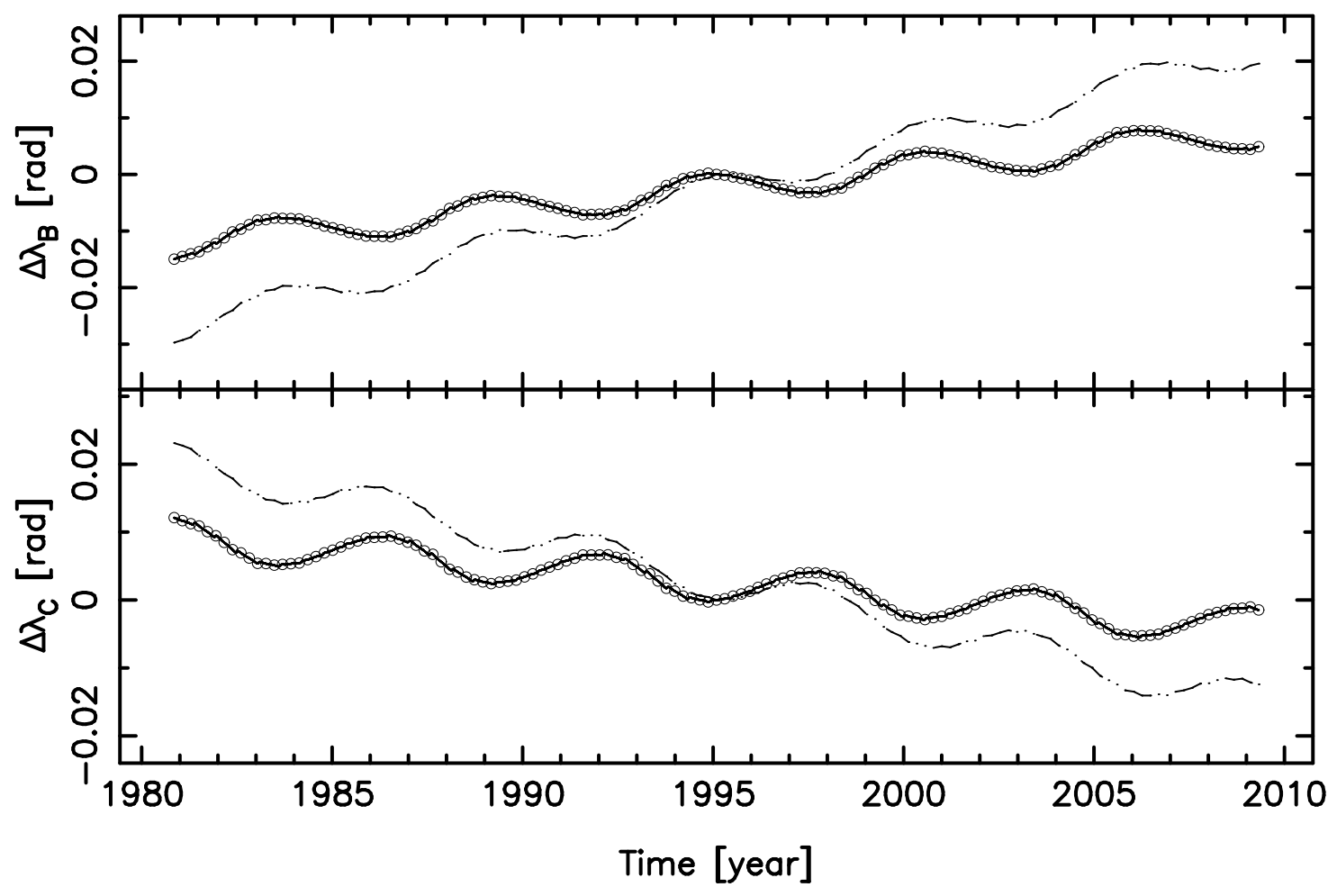

Fig. 2.- 

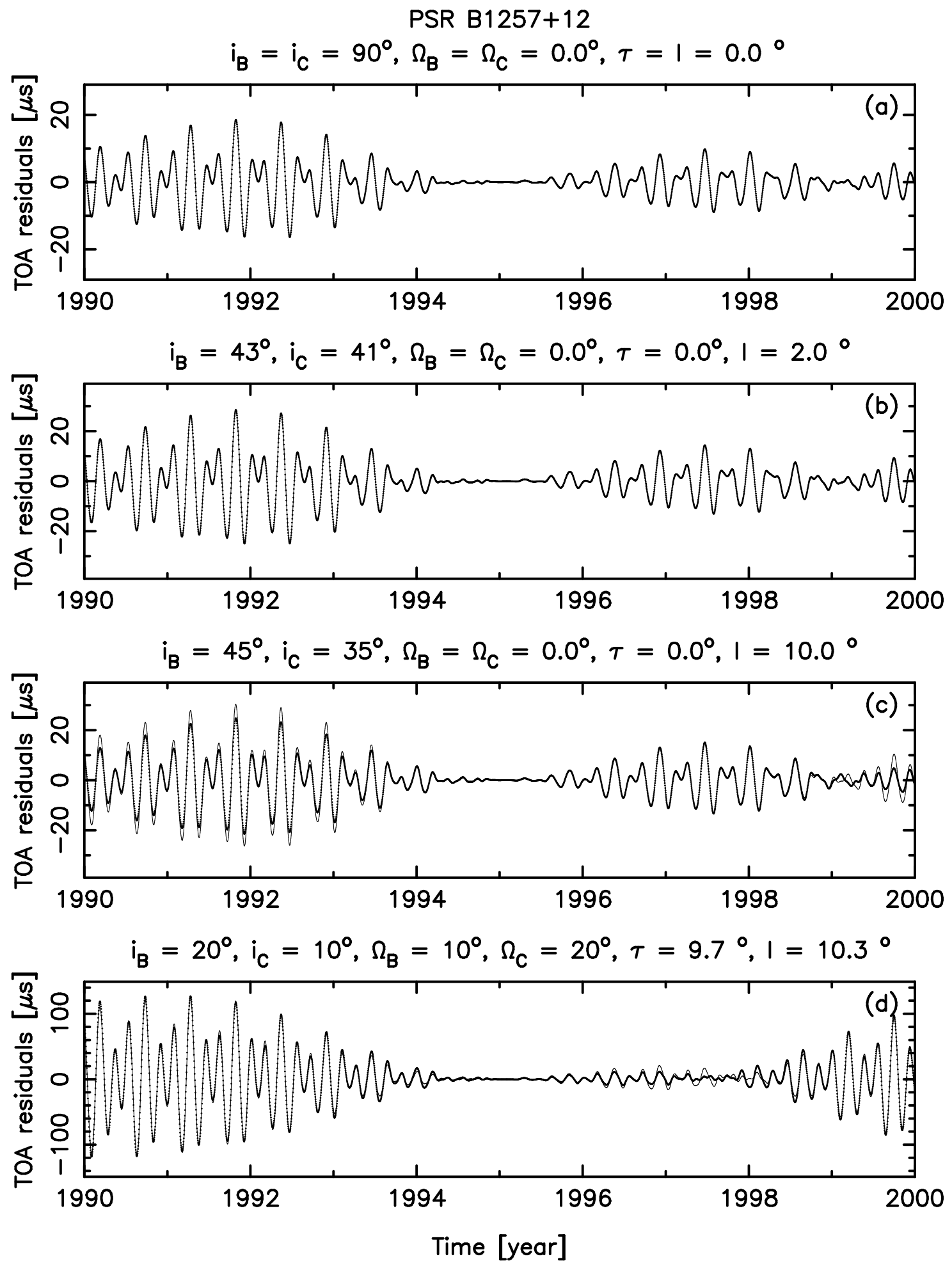

Fig. 3.- 